\title{
ALGEBRAIC INDEPENDENCE OF LOCAL CONJUGACIES AND RELATED QUESTIONS IN POLYNOMIAL DYNAMICS
}

\author{
KHOA D. NGUYEN
}

(Communicated by Matthew A. Papanikolas)

\begin{abstract}
Let $K$ be an algebraically closed field of characteristic 0 and $f \in K[t]$ a polynomial of degree $d \geq 2$. There exists a local conjugacy $\psi_{f}(t) \in t K[[1 / t]]$ such that $\psi_{f}\left(t^{d}\right)=f\left(\psi_{f}(t)\right)$. It has been known that $\psi_{f}$ is transcendental over $K(t)$ if $f$ is not conjugate to $t^{d}$ or a constant multiple of the Chebyshev polynomial. In this paper, we study the algebraic independence of $\psi_{f_{1}}, \ldots, \psi_{f_{n}}$ using a recent result of Medvedev and Scanlon. Related questions in transcendental number theory and canonical heights in arithmetic dynamics are also discussed.
\end{abstract}

\section{INTRODUCTION AND MAIN RESULTS}

Throughout this paper, let $K$ be an algebraically closed field of characteristic 0 . Let $f \in K[t]$ be a polynomial of degree $d \geq 2$. There exists a Laurent series

$$
\psi_{f}(t)=a_{-1} t+a_{0}+\frac{a_{1}}{t}+\frac{a_{2}}{t^{2}}+\ldots \in t K[[1 / t]], a_{-1} \neq 0,
$$

such that

$$
\psi_{f}\left(t^{d}\right)=f\left(\psi_{f}\right)
$$

Moreover, another series $\tilde{\psi}_{f}$ satisfies the above conditions if and only if there exists a $(d-1)$ th root of unity $\zeta$ such that $\tilde{\psi}_{f}(t)=\psi_{f}(\zeta t)$. To prove these claims, we may use the functional equation (11) to either write down and solve the system of equations involving the coefficients of $\psi_{f}$, or notice that these coefficients belong to the algebraic closure $F$ of the field generated by the coefficients of $f$, and embed $F$ into $\mathbb{C}$. Over $\mathbb{C}$, we have classical results in the complex dynamics of polynomials (see, for example, Mil06, p. 98]) which motivate the results of this paper. In fact, the inverse $\psi_{f}^{-1} \in t K[[1 / t]]$, which is well-defined up to multiplication by a $(d-1)$ th root of unity, is called the Böttcher coodinate of $f$.

The Chebyshev polynomial $C_{d}(t)$ is defined to be the unique polynomial of degree $d$ such that $C_{d}\left(t+\frac{1}{t}\right)=t^{d}+\frac{1}{t^{d}}$. When $f(t)=t^{d}$ or $f(t)=C_{d}(t)$, we may choose $\psi_{f}(t)=t$, or $\psi_{f}(t)=t+\frac{1}{t}$ respectively. When $f(t)=-C_{d}(t)$, we pick any $\mu \in K$ such that $\mu^{d-1}=-1$. Then we may choose $\psi_{f}(t)=\frac{t}{\mu}+\frac{\mu}{t}$. Therefore, if $f$ is conjugate to $t^{d}$ or $\pm C_{d}(t)$, we have that $\psi_{f} \in K(t)$.

Received by the editors October 8, 2013.

2010 Mathematics Subject Classification. Primary 11J91, 37F10; Secondary 37P30.

Key words and phrases. Local conjugacies, algebraic independence, Böttcher coordinates, canonical heights. 
Following Medvedev and Scanlon [MS14, Fact 2.25], the polynomial $f$ is said to be disintegrated if $f$ is not conjugate to $t^{d}$ or $\pm C_{d}(t)$. In BB93, Becker and Bergweiler prove that $\psi_{f}$ is transcendental over $K(t)$ if $f$ is disintegrated. Shortly after that, they prove the stronger conclusion that $\psi_{f}$ is hypertranscendental [BB95]. On the other hand, it is a well-known result in polynomial dynamics that for every disintegrated polynomial $f$ and $g$, there is a choice of $\psi_{f}$ and $\psi_{g}$ such that $\psi_{f}=\psi_{g}$ if and only if $f$ and $g$ have a common iterate.

We include a proof of this due to the lack of an immediate reference. Let $d$ and $e$ denote the degrees of $f$ and $g$ respectively. By using (1), it is easy to prove that if $f$ and $g$ have a common iterate, then we can make the choice $\psi_{f}=\psi_{g}$. Now assume that $\psi_{f}(t)=\psi_{g}(t)$; we have $\psi_{g}\left(t^{d}\right)=\psi_{f}\left(t^{d}\right)=f \circ \psi_{f}(t)$. Therefore

$$
\psi_{g}\left(t^{d e}\right)=g \circ \psi_{g}\left(t^{d}\right)=g \circ f \circ \psi_{f}(t) .
$$

Interchanging $f$ and $g$, we have:

$$
\psi_{f}\left(t^{d e}\right)=f \circ g \circ \psi_{g}(t) .
$$

Therefore $g \circ f=f \circ g$. A classical theorem of Ritt [Rit23, p. 399] concludes that $f$ and $g$ have a common iterate.

We will provide a characterization of disintegrated polynomials $f$ and $g$ such that $\psi_{f}(t)$ and $\psi_{g}(t)$ are algebraically dependent over $K(t)$. Therefore, in a certain sense, the results in this paper might be regarded as a common extension to results stated in the last paragraph. First, we need the following definition from dynamics:

Definition 1.1. Let $p(t)$ and $q(t)$ be non-linear polynomials in $K[t]$. We say that $p(t)$ is semi-conjugate to $q(t)$ if there exists a non-constant polynomial $\pi(t) \in K[t]$ such that $p \circ \pi=\pi \circ q$.

In fact, it is more natural to consider algebraic dependence of $\psi_{f}\left(\alpha t^{m}\right)$ and $\psi_{g}\left(\beta t^{n}\right)$ for certain roots of unity $\alpha, \beta$ and positive integers $m, n$. For $d \geq 2$, let $\mu_{(d)}$ denote the set of all roots of unity $\zeta$ whose order is relatively prime to $d$. Write

$$
M_{d}=\left\{\zeta t^{m}: \zeta \in \mu_{(d)}, m \in \mathbb{Z}, m>0\right\} .
$$

It is not difficult to prove that $M_{d}$ is exactly the set of all non-constant polynomials commuting with an iterate of $t^{d}$. Our main results can now be formulated independently from the choices of roots of unity in the definition of $\psi_{f}$ :

Theorem 1.2. Let $f, g \in K[t]$ be disintegrated polynomials of degrees $d, e \geq 2$ respectively. There exist $u(t) \in M_{d}$ and $v(t) \in M_{e}$ such that $\psi_{f}(u(t))$ and $\psi_{g}(v(t))$ are algebraically dependent over $K(t)$ if and only if an iterate of $f$ and an iterate of $g$ are semi-conjugate to a common polynomial.

We give a similar result in the case of more than 2 polynomials under the condition that all the degrees have a common power. Our first step in proving Theorem 1.2 is to show that $\operatorname{deg}(f)$ and $\operatorname{deg}(g)$ have a common power, and then apply the following:

Theorem 1.3. Let $n \geq 2$, and let $f_{1}, \ldots, f_{n} \in K[t]$ be polynomials whose degrees have a common power $d \geq 2$. For $1 \leq i \leq n$, write $\psi_{i}=\psi_{f_{i}}$. There exist $u_{1}(t), \ldots, u_{n}(t) \in M_{d}$ such that $\psi_{1}\left(u_{1}(t)\right), \ldots, \psi_{n}\left(u_{n}(t)\right)$ are algebraic dependent over $K(t)$ if and only if there exist $1 \leq i \neq j \leq n$ such that an iterate of $f_{i}$ and an iterate of $f_{j}$ are semi-conjugate to a common polynomial. 
The organization of this paper is as follows. We first exhibit an algebraic dependence relation between $\psi_{f}(u(t))$ and $\psi_{g}(v(t))$ for some $u, v \in M_{d}$ when an iterate of $f$ and an iterate of $g$ are semi-conjugate to a common polynomial. After that we prove Theorem 1.2, and Theorem 1.3 using the main result of Medvedev and Scanlon [MS14. Then we provide an application of our results to the simplest oneparameter family of rational maps, namely the family $\left\{t^{2}+c: c \in K\right\}$. Finally, we conclude the paper by proposing some related questions in transcendental number theory and arithmetic dynamics.

\section{Constructing algebraic Dependence Relations}

Let $v_{\infty}$ denote the usual discrete valuation on the field $K((1 / t))$ of formal Laurent series in $1 / t$. We have the following useful result:

Lemma 2.1. Let $f \in K[t]$ be a polynomial of degree $d \geq 2$. Let $D \geq 1$. Let $L(t) \in K((1 / t))$ such that $v_{\infty}(L)=D$ and $L\left(t^{d}\right)=f(L(t))$. Then there exists $\psi \in K((1 / t))$ such that $v_{\infty}(\psi)=1, \psi\left(t^{d}\right)=f(\psi(t))$, and $L(t)=\psi\left(t^{D}\right)$.

Proof. There is nothing to prove when $D=1$. We may assume that $D \geq 2$. Write

$$
L(t)=c_{D} t^{D}+c_{D-1} t^{D-1}+\ldots=\sum_{i=-\infty}^{D} c_{i} t^{i} .
$$

We prove that $L(t)=\psi\left(t^{D}\right)$ for some $\psi(t) \in K((1 / t))$ by using contradiction. Let $M$ be the largest integer such that $D$ does not divide $M$ and $c_{M} \neq 0$. Write

$$
\begin{aligned}
f(t) & =b_{d} t^{d}+\ldots+b_{0}, \\
f(L(t)) & =b_{d}\left(c_{D} t^{D}+\ldots+c_{M} t^{M}+\ldots\right)^{d}+b_{d-1}\left(c_{D} t^{D}+\ldots+c_{M} t^{M}+\ldots\right)^{d-1}+\ldots
\end{aligned}
$$

By the choice of $M$, the coefficient of $t^{D(d-1)+M}$ in $f(L(t))$ is $d b_{d} c_{D}^{d-1} c_{M}$. On the other hand, the coefficient of $t^{D(d-1)+M}$ in $L\left(t^{d}\right)$ is either 0 or $c_{N}$ if $N=$ $\frac{D(d-1)+M}{d}$ is an integer. In this latter case, we have $c_{N}=0$ since $N$ is not a multiple of $D, N>M$, and the maximality of $M$. Therefore, comparing the coefficient of $t^{D(d-1)+M}$, we have

$$
d b_{d} c_{D}^{d-1} c_{M}=0
$$

Hence $c_{M}=0$, a contradiction.

Therefore $L(t)=\psi\left(t^{D}\right)$ for some $\psi \in K((1 / t)), v_{\infty}(\psi)=1$. We have

$$
f\left(\psi\left(t^{D}\right)\right)=f(L(t))=L\left(t^{d}\right)=\psi\left(t^{D d}\right) .
$$

Hence $f(\psi(t))=\psi\left(t^{d}\right)$.

Lemma 2.1 is used to prove the following:

Proposition 2.2. Let $f, g \in K[t]$ be polynomials of degree $d \geq 2$ that are semiconjugate to a common polynomial $h$. Let $\pi, \rho \in K[t]$ be non-constant such that $f \circ \pi=\pi \circ h$ and $g \circ \rho=\rho \circ h$. Then there exists a choice of $\psi_{f}$ and $\psi_{g}$ such that $\psi_{f}\left(t^{\operatorname{deg}(\pi)}\right)$ and $\psi_{g}\left(t^{\operatorname{deg}(\rho)}\right)$ are algebraically dependent over $K$.

Proof. Fix a choice of $\psi_{h}(t)$, let $L(t)=\pi\left(\psi_{h}(t)\right)$. We have that $v_{\infty}(L)=\operatorname{deg}(\pi)$, and $L\left(t^{d}\right)=f(L(t))$ thanks to the functional equation $f \circ \pi=\pi \circ h$. By Lemma 2.1. there is a choice of $\psi_{f}$ such that $\psi_{f}\left(t^{\operatorname{deg}(\pi)}\right)=L(t)=\pi\left(\psi_{h}(t)\right)$. Similarly, there is a 
choice of $\psi_{g}$ such that $\psi_{g}\left(t^{\operatorname{deg}(\rho)}\right)=\rho\left(\psi_{h}(t)\right)$. Therefore $\psi_{f}\left(t^{\operatorname{deg}(\pi)}\right)$ and $\psi_{g}\left(t^{\operatorname{deg}(\rho)}\right)$ are algebraically dependent over $K$ since both are in the field $K\left(\psi_{h}\right)$.

Corollary 2.3. Let $f, g \in K[t]$ such that for some positive integers $n, m$, there exist non-constant $\pi, \rho \in K[t]$ and a polynomial $h \in K[t]$ of degree $\delta \geq 2$ satisfying $f^{n} \circ \pi=\pi \circ h$ and $g^{m} \circ \rho=\rho \circ h$. Fix a choice of $\psi_{f}$ and $\psi_{g}$. Then there exist $(\delta-1)$ th roots of unity $\alpha, \beta$ such that $\psi_{f}\left((\alpha t)^{\operatorname{deg}(\pi)}\right)$ and $\psi_{g}\left((\beta t)^{\operatorname{deg} \rho}\right)$ are algebraically dependent over $K$.

Proof. By Proposition 2.2, there is a choice of $\psi_{f^{m}}$ and $\psi_{g^{n}}$ such that $\psi_{f^{m}}\left(t^{\operatorname{deg}(\pi)}\right)$ and $\psi_{g^{n}}\left(t^{\operatorname{deg}(\rho)}\right)$ are algebraically dependent over $K$. Since $\psi_{f}$ and $\psi_{g}$ are other choices for $\psi_{f^{m}}$ and $\psi_{g^{n}}$ respectively, there exist $(\delta-1)$ th roots of unity $\alpha$ and $\beta$ such that $\psi_{f^{m}}(t)=\psi_{f}(\alpha t)$ and $\psi_{g^{n}}(t)=\psi_{g}(\beta t)$. This finishes the proof.

Remark 2.4. Note that Proposition 2.2 and Corollary 2.3 provide algebraic dependence over $K$, not just $K(t)$.

\section{Proof of Theorem 1.3}

If an iterate of $f_{i}$ and an iterate of $f_{j}$ are semi-conjugate to a common polynomial, then we apply Corollary 2.3 (see Remark 2.4). It remains to prove the "only if" part. Replace $d$ by an appropriate power such that each $u_{i}(t)$ commutes with $t^{d}$. Replacing each $f_{i}$ by an iterate, we may assume that all the polynomials $f_{1}, \ldots, f_{n}$ have a common degree $d$. By relabeling and removing the $f_{i}$, and lowering $n$ if necessary, we may assume that $\psi_{1}\left(u_{1}(t)\right), \ldots, \psi_{n-1}\left(u_{n-1}(t)\right)$ are algebraically independent over $K(t)$ and $\psi_{n}\left(u_{n}(t)\right)$ is algebraic of degree $D \geq 1$ over $K\left(t, \psi_{1}\left(u_{1}(t)\right), \ldots, \psi_{n-1}\left(u_{n-1}(t)\right)\right)$. We have the following irreducible polynomial in $K\left[X_{0}, X_{1}, \ldots, X_{n}\right]$ :

$$
P\left(X_{0}, X_{1}, \ldots, X_{n}\right)=P_{D}\left(X_{0}, \ldots, X_{n-1}\right) X_{n}^{D}+\ldots+P_{0}\left(X_{0}, \ldots, X_{n-1}\right)
$$

such that $P\left(t, \psi_{1}\left(u_{1}(t)\right), \ldots, \psi_{n}\left(u_{n}(t)\right)\right)=0$ and $\operatorname{gcd}\left(P_{D}, \ldots, P_{0}\right)=1$. In fact, $P$ is the minimal polynomial of $\psi_{n}\left(u_{n}(t)\right)$ over

$$
K\left[X_{0}, \ldots, X_{n-1}\right] \cong K\left[t, \psi_{1}\left(u_{1}(t)\right), \ldots, \psi_{n-1}\left(u_{n-1}(t)\right)\right] .
$$

Replacing $t$ by $t^{d}$ and using the identities $\psi_{i}\left(t^{d}\right)=f_{i}\left(\psi_{i}\right)$, and $u_{i}\left(t^{d}\right)=\left(u_{i}(t)\right)^{d}$ for each $i$, we have

$$
P\left(t^{d}, f_{1}\left(\psi_{1}\left(u_{1}(t)\right)\right), \ldots, f_{n}\left(\psi_{n}\left(u_{n}(t)\right)\right)\right)=0 .
$$

Therefore, we have that $P\left(X_{0}, \ldots, X_{n}\right)$ is a factor of $P\left(X_{0}^{d}, f_{1}\left(X_{1}\right), \ldots, f_{n}\left(X_{n}\right)\right)$. Consider the self-map $\varphi$ of $\mathbb{A}^{n+1}$ given by

$$
\varphi\left(x_{0}, \ldots, x_{n}\right)=\left(x_{0}^{n}, f_{1}\left(x_{1}\right), \ldots, f_{n}\left(x_{n}\right)\right) .
$$

Let $V$ be the hypersurface defined by $P\left(X_{0}, \ldots, X_{n}\right)=0$; then we have $V \subseteq$ $\varphi^{-1}(V)$. In other words, $V$ is an invariant hypersurface in the dynamical system $\left(\mathbb{A}^{n}, \varphi\right)$. For each $1 \leq i \neq j \leq n$, let $\pi_{i, j}$ denote the projection from $\mathbb{A}^{n}$ onto the $(i, j)$-factor $\mathbb{A}^{2}$. By the main theorem of Medvedev and Scanlon MS14, Theorem 2.30], there exists $1 \leq i \neq j \leq n$ such that $\pi_{i, j}(V)$ is an invariant curve of $\mathbb{A}^{2}$ under the self-map $\left(f_{i}, f_{j}\right)$, and

$$
V=\pi_{i, j}^{-1}\left(\pi_{i, j}(V)\right) \cong \pi_{i, j}(V) \times \mathbb{A}^{n-1} .
$$

Since $X_{n}$ cannot be the only variable that appears in $P$, there are at least 2 variables appearing in $P$. Hence the invariant curve $\pi_{i, j}(V)$ cannot map to a single 
point in each factor $\mathbb{A}^{1}$ of $\mathbb{A}^{2}$. By [MS14, Proposition 2.34], the polynomials $f_{i}$ and $f_{j}$ are semi-conjugate to a common polynomial. This finishes the proof.

Remark 3.1. It follows from the proof that if $f_{1}, \ldots, f_{n}$ have the same degree $d$, $u_{1}(t), \ldots, u_{n}(t)$ commute with $t^{d}$, and $\psi_{1}\left(u_{1}(t)\right), \ldots, \psi_{n}\left(u_{n}(t)\right)$ are algebraically dependent over $K(t)$, then there exist $1 \leq i \neq j \leq n$ such that $f_{i}$ and $f_{j}$ are semi-conjugate to a common polynomial.

Remark 3.2. The arguments here can be used in a more general setting, as follows. Let $f_{0}(t), \ldots, f_{n}(t)$ be polynomials, and let $\psi_{1}(t), \ldots, \psi_{n}(t)$ be a formal series such that $\psi_{i}\left(f_{0}(t)\right)=f_{i}\left(\psi_{i}(t)\right)$ for every $1 \leq i \leq n$. Then algebraic dependence of $\psi_{1}(t), \ldots, \psi_{n}(t)$ over $K(t)$ would give rise to an invariant hypersurface of the dynamical system $\left(\mathbb{A}^{n+1}, \varphi\right)$ where $\varphi=\left(f_{0}, \ldots, f_{n}\right)$. Then the main result of Medvedev and Scanlon can be applied.

\section{Proof of Theorem 1.2}

The "if" part follows immediately from Corollary 2.3. Before proving the "only if" part, we prove the following theorem which will also be needed later. We let $\tilde{f}$ denote a polynomial of smallest degree such that $f$ is an iterate of $\tilde{f}$. Write $\delta=\operatorname{deg}(\tilde{f})$. It is well-known that every polynomial commuting with an iterate of $f$ must have the form $\tilde{f}^{n} \circ \ell$, where $\ell$ is a linear polynomial commuting with an iterate of $f$ (see, for example, Ngu14, Proposition 2.3] and Ngu14, p. 12]). Therefore, the degree of every such polynomial is a power of $\delta$.

Theorem 4.1. Assume $n \geq 2$, and $\alpha_{1}(t), \ldots, \alpha_{n}(t) \in M_{d}(t)$ such that the functions $\psi_{f}\left(\alpha_{1}(t)\right), \ldots, \psi_{f}\left(\alpha_{n}(t)\right)$ are algebraically dependent over $K(t)$. Then there exist $1 \leq i \neq j \leq n$ such that $\frac{\operatorname{deg}\left(\alpha_{i}\right)}{\operatorname{deg}\left(\alpha_{j}\right)}$ has the form $\delta^{m}$ for some positive integer $m$.

Proof. Replace $f$ by an iterate, and hence $d$ by its power such that $\alpha_{i}(t)$ commutes with $t^{d}$ for every $1 \leq i \leq n$. As in the proof of Theorem 1.3 in the previous section, we may assume that $\psi_{f}\left(\alpha_{1}\right), \ldots, \psi_{f}\left(\alpha_{n-1}\right)$ form a transcendence basis of $K\left(t, \psi_{f}\left(\alpha_{1}\right), \ldots, \psi_{f}\left(\alpha_{n}\right)\right)$ over $K(t)$, and let $P\left(X_{0}, \ldots, X_{n}\right)$ be the minimal polynomial of $\psi_{f}(\beta)$ over $K\left[X_{0}, \ldots, X_{n-1}\right] \cong K\left[t, \psi_{f}\left(\alpha_{1}\right), \ldots, \psi_{f}\left(\alpha_{n-1}\right)\right]$. As before, we have that the hypersurface $V$ in $\mathbb{A}^{n+1}$ defined by $P\left(X_{0}, \ldots, X_{n}\right)=0$ is invariant under the self-map

$$
\varphi\left(x_{0}, x_{1}, \ldots, x_{n}\right):=\left(x_{0}^{d}, f\left(x_{1}\right), \ldots, f\left(x_{n}\right)\right) .
$$

By Theorem 2.30 and Theorem 6.24 of Medvedev and Scanlon [MS14, there exists $1 \leq i \neq j \leq n$ such that $P\left(X_{0}, X_{1}, \ldots, X_{n}\right)$ is $X_{i}-Q\left(X_{j}\right)$, where $Q$ is a polynomial commuting with $f$. Therefore we must have $\psi_{f}\left(\alpha_{i}\right)=Q\left(\psi_{f}\left(\alpha_{j}\right)\right)$. Applying the valuations $v_{\infty}$, we have $\operatorname{deg}\left(\alpha_{i}\right)=\operatorname{deg}(Q) \operatorname{deg}\left(\alpha_{j}\right)$. Therefore

$$
\frac{\operatorname{deg}\left(\alpha_{i}\right)}{\operatorname{deg}\left(\alpha_{j}\right)}=\operatorname{deg}(Q)
$$

is a power of $\delta$.

Remark 4.2. In a sense, Theorem 4.1 provides a complement to Theorem 1.3 by treating the case $f_{1}=\ldots=f_{n}$. 
We can now prove Theorem 1.2 as follows. By Theorem 1.3 , it suffices to show that $d=\operatorname{deg}(f)$ and $e=\operatorname{deg}(g)$ have a common power. Replacing $f$ and $g$ by appropriate iterates so that $d$ and $e$ are replaced by the corresponding powers, we may assume that $u(t)$ and $v(t)$, respectively, commute with $t^{d}$ and $t^{e}$. Since $\psi_{f}(u(t))$ and $\psi_{g}(v(t))$ are algebraically dependent over $K(t)$, so are $\psi_{f}\left(u\left(t^{e}\right)\right)$ and $\psi_{g}\left(v\left(t^{e}\right)\right)$. Since $\psi_{g}\left(v\left(t^{e}\right)\right)=g\left(\psi_{g}(v(t))\right)$ and $\psi_{f}(u(t))$ are algebraically dependent over $K(t)$, so are $\psi_{f}\left(u\left(t^{e}\right)\right)$ and $\psi_{f}(u(t))$. By Theorem 4.1, $e=\frac{\operatorname{deg}\left(u\left(t^{e}\right)\right)}{\operatorname{deg}(u(t))}$ has a common power with $d$. This finishes the proof of Theorem 1.2

\section{An EXAMPLE}

Our main results are useful since they can be combined to the classification of semi-conjugate polynomials given in the appendix of Inou's paper Ino11, Appendix A] using results of Ritt Rit22 and Engstrom Eng41. For example, we consider the simplest one-parameter family of rational maps: $f_{c}(t)=t^{2}+c, c \in K$. Write $\psi_{c}(t)=\psi_{f_{c}}(t)$. We have:

Corollary 5.1. Elements of $\left\{\psi_{c}(t): c \neq 0,-2\right\}$ are algebraically independent over $K(t)$.

Proof. Let $c \notin\{0,-2\}$; we claim that if $f_{c}(t)$ is semi-conjugate to $h(t)$, then $f_{c}$ and $h$ are conjugate to each other. Let $\pi \in K[t]$ be non-constant such that $f_{c} \circ \pi=\pi \circ h$. Replacing $\pi$ by $\pi \circ \ell$ and $h$ by $\ell^{-1} \circ h \circ \ell$ for an appropriate linear polynomial $\ell$, we may assume that $h(t)=t^{2}+\tilde{c}$, for some $\tilde{c} \in K$. We prove by contradiction that $\tilde{c}=c$, hence establishing the claim.

Assume that $\pi$ has the smallest (positive) degree such that there exists $\tilde{c} \neq c$, and $f_{c} \circ \pi=\pi \circ h$ for $h(t)=t^{2}+\tilde{c}$. From $\pi(t)^{2}+c=\pi\left(t^{2}+\tilde{c}\right)$, we have that $\pi(-t)= \pm \pi(t)$. If $\pi(-t)=-\pi(t)$, then $\operatorname{deg}(\pi)$ would be odd, hence $\operatorname{gcd}\left(\operatorname{deg}(\pi), \operatorname{deg}\left(f_{c}\right)\right)=1$. By [no11, Theorem 8], $f_{c}$ is conjugate to $t^{2}$ or $t^{2}-2$. This is impossible when $c \notin\{0,-2\}$.

Therefore $\pi(-t)=\pi(t)$. Write $\pi(t)=P\left(t^{2}\right)$. We have

$$
\left(P\left(t^{2}\right)\right)^{2}+c=P\left(t^{4}+2 \tilde{c} t^{2}+\tilde{c}^{2}\right) .
$$

Hence $f_{c} \circ P=P \circ \tilde{h}$, where $\tilde{h}(t)=(t+\tilde{c})^{2}$. Put $\ell(t)=t-\tilde{c}$ so that $\ell^{-1} \circ \tilde{h} \circ \ell=$ $t^{2}+\tilde{c}=h(t)$. Then $\tilde{\pi}=P \circ \ell$ violates the choice of $\pi$, a contradiction. This proves the claim at the beginning.

For $c, d \notin\{0,-2\}$ and $c \neq d$, the polynomials $f_{c}$ and $f_{d}$ are not conjugate to each other. Therefore, they cannot be semi-conjugate to a common polynomial by the claim at the beginning. By Remark 3.1 the functions $\left\{\psi_{c}: c \neq 0,-2\right\}$ are algebraically independent over $K(t)$.

\section{Some Related questions}

6.1. More than two polynomials. We expect Theorem 1.3 still holds without the extra restriction on the degrees. In other words, Theorem 1.2 holds for more than two polynomials. However, it does not seem to follow easily from the arguments given in this paper. 
6.2. Mahler's method and algebraic independence of values. For the rest of this paper, we assume $K=\overline{\mathbb{Q}}$ with a fixed embedding into $\mathbb{C}$. Becker and Bergweiler [BB93] also prove that for every disintegrated $f$, for every $a \in \overline{\mathbb{Q}} \subset \mathbb{C}$ such that $|a|$ is sufficiently large, $\psi_{f}(a)$ is transcendental. Their proof uses "Mahler's method", in which the transcendence of $\psi_{f}$ is the first important step so that certain auxiliary polynomials can be constructed (see [Mah29] or [Nis96, Chapter 1]).

Certain attempts have been made to prove algebraic independence of values of "Mahler's functions" $f_{1}, \ldots, f_{n}$ for $n \geq 2$. As far as we know, only the case of linear functional equations has been treated by various authors (see [Nis96. Chapter 3] and the references there). More precisely, they consider functions $f_{1}(t), \ldots, f_{n}(t)$ such that

$$
f_{i}\left(t^{d}\right)=a_{i, 1}(t) f_{1}(t)+\ldots+a_{i, n} f_{n}(t)+b_{i}(t)
$$

for some $a_{i, 1}, \ldots, a_{i, n}, b_{i} \in \overline{\mathbb{Q}}(t)$, for every $1 \leq i \leq n$.

We may ask the following interesting question:

Question 6.1. Let $f_{1}, \ldots, f_{n}$ be disintegrated polynomials of a common degree $d \geq 2$. Write $\psi_{i}=\psi_{f_{i}}$ for $1 \leq i \leq n$. Assume that for every $1 \leq i \neq j \leq n$, for every $N \geq 1$, the iterates $f_{i}^{N}$ and $f_{j}^{N}$ are not semi-conjugate to a common polynomial. Is it true that for every $a \in \overline{\mathbb{Q}}$ such that $|a|$ is sufficiently large, the numbers $\psi_{1}(a), \ldots, \psi_{n}(a)$ are algebraically independent over $\mathbb{Q}$ ?

Theorem 1.3 shows that $\psi_{1}, \ldots, \psi_{n}$ are algebraically independent over $K(t)$. We hope experts in Mahler's method can somehow utilize our result as a first step in answering Question 6.1.

6.3. Böttcher coordinates, Green's functions and canonical heights. The Böttcher coordinate of $f$ is the inverse $\phi_{f}(t)=\psi_{f}^{-1}(t)$ which is well-defined up to multiplication by a root of unity [Mil06, Chapter 9]. We may ask the following question:

Question 6.2. Let $f_{1}, \ldots, f_{n}$ be disintegrated polynomials of degrees at least 2 . Write $\phi_{i}=\phi_{f_{i}}$ for $1 \leq i \leq n$.

(a) Give a necessary and sufficient condition such that $\phi_{1}, \ldots, \phi_{n}$ are algebraically independent over $\overline{\mathbb{Q}}(t)$.

(b) Suppose $\phi_{1}, \ldots, \phi_{n}$ are algebraically independent over $\overline{\mathbb{Q}}(t)$. Is it true that $\phi(a), \ldots, \phi_{n}(a)$ are algebraically independent over $\overline{\mathbb{Q}}$ for every $a \in \overline{\mathbb{Q}}$ such that $|a|$ is sufficiently large?

For every polynomial $f$ of degree $d \geq 2$, the dynamical Green's function $G_{f}$ is defined as

$$
G_{f}(a)=\lim _{n \rightarrow \infty} \frac{\log \left|f^{n}(a)\right|}{d^{n}} \forall a \in \mathbb{C} .
$$

One could show that $G_{f}(a)=\log \left|\phi_{f}(a)\right|$ for all sufficiently large $|a|$ Mil06, Chapter 9]. We conclude this paper by explaining the relation between transcendence of values of Green's functions and irrationality of values of canonical heights.

Let $h$ denote the absolute logarithmic height on $\mathbb{P}^{1}(\overline{\mathbb{Q}})$ [Sil07, Chapter 3]. For every rational map $\varphi \in \overline{\mathbb{Q}}(t)$ of degree $d \geq 2$, the canonical height $\hat{h}_{\varphi}$ is defined as

$$
\hat{h}_{\varphi}(a)=\lim _{n \rightarrow \infty} \frac{h\left(\varphi^{n}(a)\right)}{d^{n}} \forall a \in \overline{\mathbb{Q}} .
$$


It is not difficult to prove that $a$ is $\varphi$-preperiodic if and only if $\hat{h}_{\varphi}(a)=0$ Sil07, Chapter 3]. Silverman suggests the following question:

Question 6.3. Is it true that for every $\varphi$ and for every $\varphi$-wandering $a$, the number $\hat{h}_{\varphi}(a)$ is transcendental?

As far as we know, almost nothing is known about Question 6.3. In fact, not much is known about the following much weaker:

Question 6.4. Let $f \in \mathbb{Q}[t]$ be a polynomial of degree $d \geq 2$. Is it true that there exists $a \in \mathbb{Q}$ such that $\hat{h}_{f}(a)$ is irrational?

For $f$ as in Question 6.4, and for every $a \in \mathbb{Q}$, we have the following:

$$
\hat{h}_{f}(a)=G_{f}(a)+\log \left|R_{f}(a)\right|,
$$

where $R_{f}(a) \in \mathbb{Q}$ is the "contribution from the finite primes" which is relatively easy to handle [Sil07, Chapter 5]. Let $K_{f}$ denote the filled Julia set of $f$ [Mil06, Chapter 9]. If $K_{f} \cap \mathbb{R}$ has a non-empty interior, one can pick any $f$-wandering $b \in K_{f} \cap \mathbb{Q}$ so that $G_{f}(b)=0$; hence $\hat{h}_{f}(b)=\log \left|R_{f}(b)\right|$ is irrational (indeed transcendental by the Lindemann-Weierstrass theorem). In general when we cannot pick any $f$-wandering $b$ from $K_{f} \cap \mathbb{Q}$; hence the difficulty comes from the presence of the archimedean contribution $G_{f}$.

Pick a choice of $\phi_{f}$. Let $\bar{\phi}_{f}$ denote the Laurent series obtained from $\phi_{f}$ by taking the complex conjugate of each coefficient. Since $f(t) \in \mathbb{Q}[t]$, we have $\bar{\phi}_{f}(f(t))=$ $\left(\bar{\phi}_{f}(t)\right)^{d}$. Hence there exists a $(d-1)$ th root of unity $\zeta$ such that $\bar{\phi}_{f}=\zeta \phi_{f}$. For every $a \in \mathbb{Q}$ such that $|a|$ is sufficiently large, we have

$$
\begin{aligned}
G_{f}(a)=\log \left|\phi_{f}(a)\right| & =\frac{1}{2} \log \left(\phi_{f}(a) \bar{\phi}_{f}(a)\right)=\frac{1}{2} \log \zeta\left(\phi_{f}(a)\right)^{2} \\
& =\frac{1}{2(d-1)} \log \left(\phi_{f}(a)\right)^{2(d-1)} .
\end{aligned}
$$

Therefore, we can rewrite (2) as

$$
\hat{h}_{f}(a)=\frac{1}{2(d-1)} \log \phi_{f}(a)^{2(d-1)}+\log \left|R_{f}(a)\right| .
$$

Let $g \in Q[t]$ be another polynomial. If $a \in \mathbb{Q}$ such that $|a|$ is sufficiently large, and $\frac{\hat{h}_{f}(a)}{\hat{h}_{g}(a)} \in \mathbb{Q}$, then (3) implies that there exist positive integers $m$ and $n$ such that $\frac{\phi_{f}(a)^{m}}{\phi_{g}(a)^{n}} \in \mathbb{Q}$. Therefore, we have the following:

Remark 6.5. Suppose that part (b) of Question 6.2 has an affirmative answer. Let $f, g \in \mathbb{Q}[t]$ be disintegrated polynomials of degree at least 2 such that $\phi_{f}$ and $\phi_{g}$ are algebraically independent over $\overline{\mathbb{Q}}(t)$. Then for every $a \in \mathbb{Q}$ such that $|a|$ is sufficiently large, we have that $\frac{\hat{h}_{f}(a)}{\hat{h}_{g}(a)}$ is irrational.

\section{ACKNOWLEDGMENTS}

The author would like to thank Tom Tucker, Tom Scanlon, and the anonymous referee for many useful suggestions. We are grateful to Patrick Ingram and Joe Silverman for helpful conversations involving Section 6 . 


\section{REFERENCES}

[BB93] Paul-Georg Becker and Walter Bergweiler, Transcendency of local conjugacies in complex dynamics and transcendency of their values, Manuscripta Math. 81 (1993), no. 3-4, 329337, DOI 10.1007/BF02567862. MR1248759 (94i:11051)

[BB95] Paul-Georg Becker and Walter Bergweiler, Hypertranscendency of conjugacies in complex dynamics, Math. Ann. 301 (1995), no. 3, 463-468, DOI 10.1007/BF01446639. MR 1324520 (96g:30052)

[Eng41] H. T. Engstrom, Polynomial substitutions, Amer. J. Math. 63 (1941), 249-255. MR0003599(2,242f)

[Ino11] Hiroyuki Inou, Extending local analytic conjugacies, Trans. Amer. Math. Soc. 363 (2011), no. 1, 331-343, DOI 10.1090/S0002-9947-2010-05049-4. MR2719684 (2012h:37093)

[Mah29] Kurt Mahler, Arithmetische Eigenschaften der Lösungen einer Klasse von Funktionalgleichungen (German), Math. Ann. 101 (1929), no. 1, 342-366, DOI 10.1007/BF01454845. MR.1512537

[Mil06] John Milnor, Dynamics in one complex variable, 3rd ed., Annals of Mathematics Studies, vol. 160, Princeton University Press, Princeton, NJ, 2006. MR2193309 (2006g:37070)

[MS14] Alice Medvedev and Thomas Scanlon, Invariant varieties for polynomial dynamical systems, Ann. of Math. (2) 179 (2014), no. 1, 81-177, DOI 10.4007/annals.2014.179.1.2. MR3126567

[Ngu14] K. Nguyen, Some arithmetic dynamics of diagonally split polynomial maps, to appear in Int. Math. Res. Not. IMRN. arXiv:1304.3052v2, 2014.

[Nis96] Kumiko Nishioka, Mahler functions and transcendence, Lecture Notes in Mathematics, vol. 1631, Springer-Verlag, Berlin, 1996. MR1439966 (98d:11084)

[Rit22] J. F. Ritt, Prime and composite polynomials, Trans. Amer. Math. Soc. 23 (1922), no. 1, 51-66, DOI 10.2307/1988911. MR.1501189

[Rit23] J. F. Ritt, Permutable rational functions, Trans. Amer. Math. Soc. 25 (1923), no. 3, 399-448, DOI 10.2307/1989297. MR:1501252

[Sil07] Joseph H. Silverman, The arithmetic of dynamical systems, Graduate Texts in Mathematics, vol. 241, Springer, New York, 2007. MR2316407 (2008c:11002)

Department of Mathematics, University of California, Berkeley, California 94720 E-mail address: khoanguyen2511@gmail.com

$U R L:$ www.math. berkeley.edu/ ${ }^{k h o a}$

Current address: Department of Mathematics, University of British Columbia, and Pacific

Institute for the Mathematical Sciences, Vancouver, British Columbia V6T 1Z2, Canada

E-mail address: dknguyen@math.ubc.ca

$U R L$ : www.math.ubc.ca/ ${ }^{\text {dknguyen }}$ 